\title{
Clinical Correlate of Brain SPECT Perfusion Abnormalities in Fibromyalgia
}

\author{
Eric Guedj ${ }^{1}$, Serge Cammilleri ${ }^{1}$, Jean Niboyet ${ }^{2}$, Patricia Dupont ${ }^{2}$, Eric Vidal ${ }^{2}$, Jean-Pierre Dropinski ${ }^{2}$, \\ and Olivier Mundler ${ }^{1}$ \\ ${ }^{I}$ Service Central de Biophysique et de Médecine Nucléaire, AP-HM Timone, Marseille, France; and ${ }^{2}$ Unité d'Etude et de Traitement de la \\ Douleur, Clinique La Phocéanne, Marseille, France
}

\begin{abstract}
The purpose of this study was to investigate the specific clinical correlate of brain SPECT perfusion abnormalities reported in fibromyalgia. Methods: We performed a whole-brain voxel-based correlation analysis involving regional cerebral blood flow and various parameters related to pain (Visual Analog Scale, Tubingen Pain Behavior Scale, and Questionnaire Douleur de Saint-Antoine Scale), disability (Fibromyalgia Impact Questionnaire [FIQ]), and anxiety and depression status (Hospital Anxiety and Depression scale) in 20 patients with fibromyalgia ( $P$ voxel $<$ 0.005). Ten healthy control women were also included, in order to determine areas of significant hypo- and hyperperfusions in patients. Results: FIQ total score was positively correlated with bilateral parietal perfusion, including postcentral cortex. These clusters of correlation were included in the areas of significant hyperperfusion. FIQ total score was also negatively correlated with perfusion of a left anterior temporal cluster, included in the areas of significant hypoperfusions. No other clinical correlation was observed with regional cerebral blood flow. Conclusion: These results show that brain perfusion abnormalities in patients with fibromyalgia are correlated with the clinical severity of the disease.
\end{abstract}

Key Words: brain SPECT; voxel-based analysis; fibromyalgia; pain; disability

J Nucl Med 2008; 49:1798-1803

DOI: 10.2967/jnumed.108.053264

$\mathbf{F}$ ibromyalgia is considered to be one of the most common causes of musculoskeletal pain and disability (1). This syndrome may be related to a global dysfunction of cerebral pain processing, notably involving central sensitization (2). This pathophysiologic mechanism also has been involved in psychologic or psychiatric symptoms of fibromyalgia. The relationship between these symptoms and fibromyalgia is bidirectional. Psychologic or psychiatric symptoms may antedate and play a causative role in the

\footnotetext{
Received Apr. 8, 2008; revision accepted Jul. 23, 2008.

For correspondence or reprints contact: Eric Guedj, Service Central de Biophysique et de Médecine Nucléaire, Assistance Publique des Hôpitaux de Marseille, Centre Hospitalo-Universitaire de la Timone, 264 Rue Saint Pierre, 13385 Marseille Cedex 5, France.

E-mail: eric.guedj@ap-hm.fr

COPYRIGHT @ 2008 by the Society of Nuclear Medicine, Inc.
}

onset of pain and disability or may follow the development of fibromyalgia (3).

We recently showed that patients with fibromyalgia exhibit significant brain ${ }^{99 \mathrm{~m}}$ Tc-ethylcysteinate dimer SPECT perfusion abnormalities including, in particular, hyperperfusion of the somatosensory cortex and hypoperfusion in the frontal, cingulate, medial temporal, and cerebellar cortices (4). The SPECT findings concur with the supposed increase in nociceptive perception and decrease in affectiveattentional response to pain. However, the significance of these abnormalities remains unclear, especially because functional cortical impairment has been inconsistently reported at baseline in patients with fibromyalgia (5-8).

The objective of the present study was to investigate the specific clinical correlate of these functional abnormalities. We performed a whole-brain voxel-based analysis to determine the relationship between regional cerebral blood flow (rCBF) and clinical parameters related to pain, disability, and anxiety and depression status (9-12). These cerebral areas of correlation were compared with areas of significant hypo- and hyperperfusion found through comparisons with healthy subjects.

\section{MATERIALS AND METHODS}

\section{Patients and Control Subjects}

Twenty women with fibromyalgia (mean age \pm SD, $48.4 \pm$ 10.6 y [range, 25-63 y]) who fulfilled the American College of Rheumatology criteria (9) were consecutively enrolled in the study. All patients underwent a general medical assessment by 1 investigator to confirm the diagnosis. Exclusion criteria included pregnancy and concomitant major medical or psychiatric disease. No patient was receiving treatment with a strong opioid or $N$-methyl-D-aspartate receptor antagonist. Data on patients and healthy controls are presented in Table 1.

Pain intensity was evaluated by a Visual Analog Scale (VAS), the Questionnaire Douleur de Saint-Antoine scale (QDSA; the validated French version of the McGill Pain Questionnaire) (10), and the Tubingen Pain Behavior Scale (TBS) (11). QDSA measures the different components of the subjective pain experience, describing the sensory and the affective dimensions. The higher the QDSA score is, the worse is the condition. The TBS, evaluated by 1 investigator, rates the presence of 11 pain behaviors (e.g., 
TABLE 1

Characteristics of Subjects

\begin{tabular}{lcc}
\hline \multicolumn{1}{c}{ Characteristic } & $\begin{array}{c}\text { Patients with } \\
\text { fibromyalgia }\end{array}$ & $\begin{array}{c}\text { Healthy } \\
\text { controls }\end{array}$ \\
\hline$n$ & 20 & 10 \\
Age (y) & $48.4 \pm 10.6$ & $52.0 \pm 7.0$ \\
VAS for pain & $81.2 \pm 6.2$ & 0 \\
QDSA & & \\
$\quad$ Sensory score & $6.9 \pm 1.9$ & 0 \\
$\quad$ Affective score & $7.4 \pm 2.3$ & 0 \\
TBS score & $6.3 \pm 1.5$ & 0 \\
FIQ total score & $66.6 \pm 12.9$ & - \\
HAD & $22.7 \pm 7.8$ & $7.0 \pm 2.3$ \\
$\quad$ Total score & $12.0 \pm 5.4$ & $4.6 \pm 1.9$ \\
$\quad$ Anxiety subscore & $10.7 \pm 3.5$ & $2.4 \pm 2.3$ \\
Depression subscore & & \\
\hline
\end{tabular}

Data are mean \pm SD.

groaning, slowed movements, pain-based refusal of activities) on a 0-2 scale (0, none; 1 , sometimes; and 2, always).

Disability was evaluated using the Fibromyalgia Impact Questionnaire (FIQ). The FIQ is designed to measure the severity of fibromyalgia disease symptoms. It is a standardized, selfadministered instrument that consists of visual analog scales and questions regarding limitations on activities of daily living. The FIQ evaluates the components of health status that are believed to be most affected by fibromyalgia. This scale comprises 20 items that assess 10 subheadings. The first 11 items make up a physical impairment scale. Each item is scored on a 4-point rating scale. Items 12 and 13 ask patients to mark the number of days they felt well and the number of days they were unable to work because of fibromyalgia symptoms. Items $14-20$ are $10-\mathrm{cm}$ visual analog scales, marked in $1-\mathrm{cm}$ increments, on which the patient rates occupational difficulty, pain, fatigue, morning tiredness, stiffness, anxiety, and depression. Questions 1-13 are normalized to 10 points, and the total score is added to the number of points from the remaining questions. The total score of the FIQ ranges from 0 to 100 , and the higher the score is, the worse is the condition (i.e., the more serious the disease).

Anxiety and depression were evaluated by the Hospital Anxiety and Depression (HAD) scale score (12). HAD is a self-screening questionnaire that consists of 14 questions, 7 for anxiety and 7 for depression. A higher score means a worse condition.

For comparison of imaging findings, 10 healthy control women without any pain, who were similar in age, were also included $(52 \pm 7 \mathrm{y} ; t$ test $>0.1$; HAD subscales $<8)$. All of the subjects provided informed consent according to institutional guidelines.

\section{SPECT Protocol and Statistical Analysis}

Patients received an injection of $740 \mathrm{MBq}$ of ${ }^{99 \mathrm{~m}} \mathrm{Tc}$-ethylcysteinate dimer (Neurolite; BMS) and were kept at rest for $1 \mathrm{~h}$, in quiet surroundings, with their eyes closed. SPECT was performed using a double-head rotating $\gamma$-camera (ECAM; Siemens) equipped with a fanbeam collimator. Data were collected in 64 projections (40-s spread) through $360^{\circ}$. Tomographic 3-dimensional reconstructions were performed using a filtered backprojection algorithm and Chang attenuation correction.
A voxel-by-voxel group correlation study was performed using SPM2 (Welcome Department of Cognitive Neurology, University College, London) running on MATLAB (version 6.0; MathWorks Inc.). Images were initially converted from the DICOM to the Analyze format using MRIcro (available at www.mricro.com) and transferred to SPM2. The data were then standardized on the Montreal Neurologic Institute atlas by using a 12-parameter affine transformation, followed by nonlinear transformations and trilinear interpolation. The dimensions of the resulting voxels were $2 \times 2 \times 2$ $\mathrm{mm}$. Standardized data were then smoothed by a gaussian filter (full width at half maximum, $12 \mathrm{~mm}$ ). Correlation studies between brain perfusion and VAS for pain, FIQ total score, QDSA affective and sensory scores, and HAD total scale score and subscores were performed using a single-subject, covariates-only statistical model. Age was considered as a nuisance variable to avoid the specific effect of age on rCBF. Other clinical variables were not included as covariate variables because they were thought to characterize the disease.

Positive and negative correlations were determined. Global normalization was performed using proportional scaling. The SPM $t$ maps were initially obtained at a height threshold of $P$ less than 0.005 , corrected for multiple comparisons for the cluster $(P<$ $0.05)$. Small-volume correction was additionally used to study the clinical correlation with subcortical structures, as previously described (4). Clusters of correlation were compared with those of significant hypo- or hyperperfusion, obtained using the comparepopulations 1-scan-per-subject routine between the group with fibromyalgia and the control group, which carries out a fixedeffects, simple $t$ test for each voxel $(P<0.005$, corrected for multiple comparisons for the cluster, with age as the nuisance variable). Montreal Neurologic Institute coordinates were then converted into Talairach coordinates using the Talairach Daemon server (available at http://ric.uthscsa.edu/projects/talairachdaemon.html). Results were listed by clusters, and Brodmann area (BA), $k$ value, $t$ score, and Talairach coordinates of peak voxels were provided for each cluster. The $k$ value represents the number of significant voxels in the particular cluster. Spearman correlation was used to study the relationship between extracted clusters of perfusion and clinical variables. The $t$ test was used to compare means between subgroups, and $\mathrm{F}$ test was used to compare variance between subgroups.

\section{RESULTS}

\section{Clinical Characteristics of Subjects}

According to the inclusion criteria, healthy subjects had no pain, and their HAD subscales were less than 8. By contrast, patients with fibromyalgia exhibited pain, disability, and anxiety or depression symptoms. Clinical characteristics are presented in Table 1. In particular, HAD total score and subscores were higher in patients with fibromyalgia than in healthy subjects $(P<0.0005)$.

\section{Brain SPECT Comparison Between Patients and Control Subjects}

Compared with healthy controls, patients with fibromyalgia exhibited posterior hyperperfusion, including of the somatosensory cortex, and hypoperfusion of the frontal, cingulate, temporal, and cerebellar cortices (Fig. 1). In 


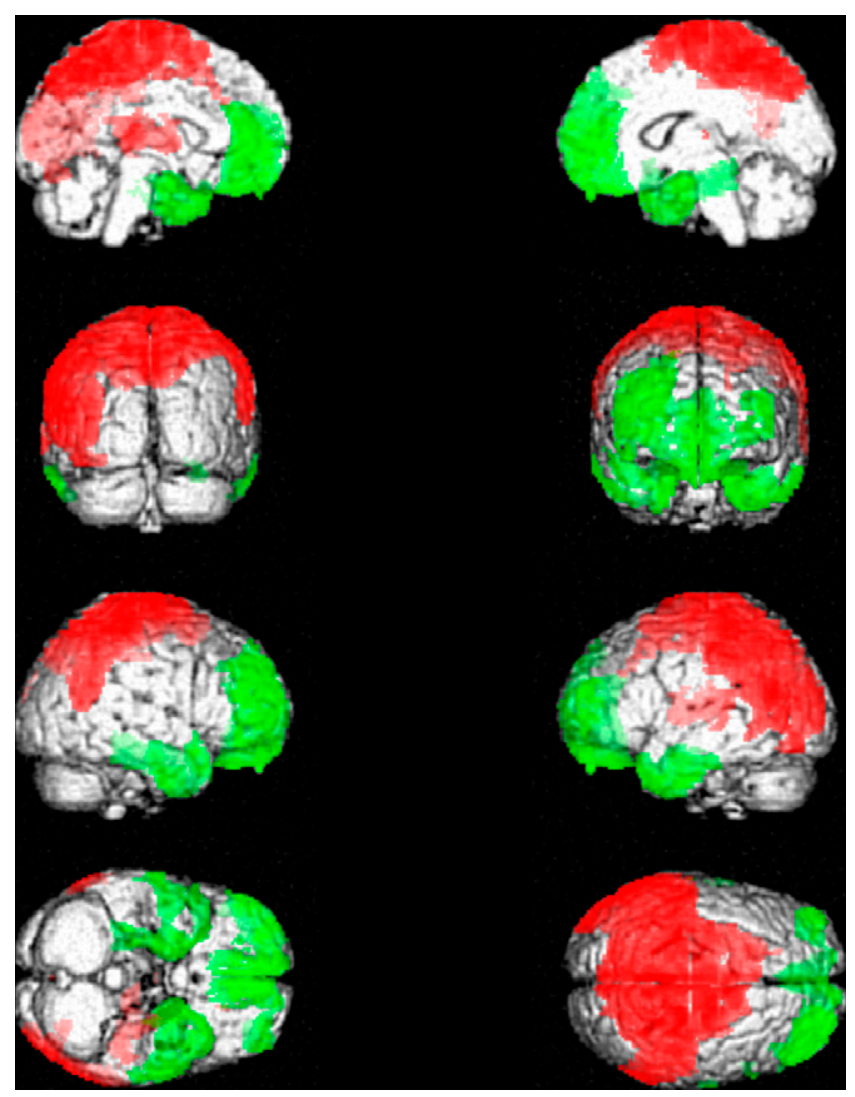

FIGURE 1. Anatomic localization of peaks of significant differences between brain SPECT of patients with fibromyalgia and healthy subjects $(P$ voxel $<0.005 ; P$ cluster $<0.05$, corrected for multiple comparisons; age as nuisance variable). Compared with healthy controls, patients with fibromyalgia exhibited posterior hyperperfusion (red), including somatosensory cortex, and hypoperfusion (green) of frontal, cingulate, temporal, and cerebellar cortices. In particular, temporal hypoperfusion included polar and mediobasal cortices. BA = Brodmann area.

particular, the temporal hypoperfusion included the polar and mediobasal cortices.

\section{Brain SPECT Perfusion Correlation}

The FIQ total score was positively correlated with perfusion of the bilateral superior parietal lobule, the bilateral precuneus (BA7), the left postcentral cortex (BA7) $(\mathrm{r}=$ 0.6544; $P=0.0043$; Talairach coordinates of the local maxima of this cluster, $x=12, y=-61$, and $z=56$ ), and the right pre- and postcentral cortex (BA4, BA6, BA1, and BA3) $(\mathrm{r}=0.5753 ; P=0.0122$; Talairach coordinates of the local maxima of this cluster, $x=42, y=-19$, and $z=43$ ) (Table 2; Fig. 2). These 2 clusters of positively correlated perfusion were included in the areas of significant hyperperfusions (respectively, $P$ cluster $=0.012$, corrected for multiple comparisons, $k=927$; and $P$ cluster $=0.038$, corrected for multiple comparisons, $k=684$ ). The FIQ total score was negatively correlated with perfusion of a left anterior temporal cluster, including the inferior, middle, and superior temporal gyri (BA21 and BA38); fusiform gyrus (BA20); and uncus (BA28 and BA36) $(\mathrm{r}=-0.5617$; $P=0.0143$; Talairach coordinates of the local maxima of this cluster, $x=-48, y=-1$, and $z=-15$ ). This cluster of negatively correlated perfusion was included in the areas of significant hypoperfusions $(P$ cluster $=0.011$, corrected for multiple comparisons, $k=908$ ).

Extracted perfusion values that positively and negatively correlated with the FIQ total score were independent of other clinical variables studied (VAS pain scale, QDSA scale, TBS, and HAD total score and subscores). FIQ total score was not correlated with other clinical variables.

No positive or negative correlations were observed between brain SPECT perfusion and other clinical variables (VAS pain scale, QDSA scale, TBS, and HAD total score and subscores), even by limiting the voxel-based analysis to significant hypo- or hyperperfusions found through comparisons with healthy subjects or by using small-volume correction for subcortical structures. In particular, no clinical correlation was observed with brain SPECT perfusion of the frontal, cingulate, and cerebellar cortices.

\section{DISCUSSION}

The FIQ is designed to measure the severity of fibromyalgia symptoms. This scale is considered to be the best measure of self-reported function and occupational disability (13). In the present study, we confirmed perfusion abnormalities previously reported in patients with fibromyalgia, compared with healthy subjects $(4,14)$, and showed that bilateral hyperperfusion of the parietal cortex and of the pre- and postcentral cortices and hypoperfusion of the left anterior temporal cortex are both strongly correlated with the FIQ total score in patients with fibromyalgia. In particular, the relationship between somatosensory hyperperfusion and fibromyalgia clinical severity is reported for, to our knowledge, the first time and reinforces the central sensitization hypothesis $(2,15)$. On the other hand, correlation obtained with anterior temporal perfusion suggests the involvement of the limbic system. Hypoperfusion of this area could be related to reduced affective appraisal and responsiveness, frequently observed in patients with fibromyalgia (15). FIQ total score has been previously correlated with hypoperfusion of the gyrus rectalis (16). This relationship, described with a volume-of-interest analysis, is consistent with our findings, because this cerebral area is anatomically and functionally close to the anterior temporal cortex (17). Similarly, Walitt et al. recently reported concomitant improvement in limbic metabolism and clinical severity (18). Interestingly, and despite a relatively high mean HAD total score in our group, correlative results obtained with brain SPECT perfusion were independent of anxiety and depressive status.

By contrast, no clinical correlation was observed with the other hypoperfusions reported, in particular with the cerebellum and the frontal and cingulate cortices (4). Impair- 
TABLE 2

Correlation Between FIQ Total Score and Brain SPECT Perfusion

\begin{tabular}{|c|c|c|c|c|c|c|c|}
\hline \multirow[b]{2}{*}{ Correlation } & \multirow{2}{*}{$\begin{array}{l}P \text { cluster } \\
\text { (corrected) }\end{array}$} & \multirow[b]{2}{*}{$k$ value } & \multirow[b]{2}{*}{ Voxel $t$ score } & \multicolumn{3}{|c|}{$\begin{array}{l}\text { Talairach coordinates } \\
\text { (mm) }\end{array}$} & \multirow[b]{2}{*}{ Region (BA) } \\
\hline & & & & $x$ & $y$ & $z$ & \\
\hline \multirow[t]{10}{*}{ Positive } & 0.025 & 996 & 5.56 & 12 & -61 & 56 & Right superior parietal lobule (7) \\
\hline & & & 5.50 & 12 & -57 & 65 & Right precuneus (7) \\
\hline & & & 4.54 & -10 & -63 & 58 & Left superior parietal lobule (7) \\
\hline & & & 3.94 & -18 & -55 & 67 & Left postcentral gyrus (7) \\
\hline & & & 3.24 & -18 & -57 & 58 & Left precuneus (7) \\
\hline & 0.010 & 1,204 & 4.59 & 42 & -19 & 43 & Right postcentral gyrus (3) \\
\hline & & & 4.50 & 46 & -16 & 39 & Right precentral gyrus (4) \\
\hline & & & 4.20 & 36 & -20 & 56 & Right precentral gyrus (4) \\
\hline & & & 3.68 & 51 & -22 & 60 & Right postcentral gyrus (1) \\
\hline & & & 3.45 & 16 & -18 & 65 & Right precentral gyrus (6) \\
\hline \multirow[t]{7}{*}{ Negative } & 0.001 & 1,730 & 6.30 & -48 & -1 & -15 & Left middle temporal gyrus (21) \\
\hline & & & 4.07 & -32 & 10 & -27 & Left superior temporal gyrus (38) \\
\hline & & & 3.79 & -32 & 1 & -22 & Left uncus (28) \\
\hline & & & 3.52 & -63 & -3 & -17 & Left inferior temporal gyrus (21) \\
\hline & & & 3.43 & -46 & -11 & -26 & Left fusiform gyrus (20) \\
\hline & & & 3.33 & -28 & -9 & -30 & Left uncus (28) \\
\hline & & & 3.09 & -24 & -3 & -30 & Left uncus (36) \\
\hline
\end{tabular}

Results are listed by clusters. $k$ value represents number of significant voxels in particular cluster.

ment of these areas is thought to be particularly related to the attentional dimension of the pain $(19,20)$. Certainly, this aspect is poorly assessed by the FIQ total score and other variables explored in the present study. Other studies will be necessary to confirm this relationship. The absence of a correlation between brain SPECT abnormalities and pain VAS may be because of the small variance of this clinical parameter in our group. On the contrary, other clinical variables showed high heterogeneity, with higher variance than FIQ $(P<0.05$ using the $\mathrm{F}$ test $)$. This discrepancy could also be responsible for the absence of a correlation between $\mathrm{rCBF}$ and other clinical variables. Our study has some additional limitations, for instance, the relatively low number of participants and the difference between the number of patients and the number of participants in the control group. The questionnaires chosen for our study are largely used in fibromyalgia; we should, however, discuss whether they are appropriate for such a use. Also, TBS was not evaluated by independent, masked investigators.

Functional cortical impairment has been inconsistently reported at baseline in patients with fibromyalgia. Subcortical abnormalities have been more largely described, but without the demonstration of a clinical correlation. Mountz et al. performed the first SPECT study of rCBF in patients with fibromyalgia and healthy subjects $(5,6)$. These patients showed significantly lower rCBF in the left and right hemithalami and the left and right caudate nuclei and also exhibited significantly lower total cortical blood flow. Functional subcortical impairment in patients with fibromyalgia was then confirmed using voxel-by-voxel analysis by Kwiatek et al., with hypoperfusion of the right thalamus, right lentiform nucleus, and bilateral pontine tegmentum and no cortical abnormalities (7). No hyperperfusion was described in these 2 studies, and no clinical correlation was found with SPECT findings. By contrast, Gur et al. described both hypoperfusion and clinical correlation using a volume-of-interest analysis (16). This study found bilateral hyperperfusion of the caudate nucleus and hypoperfusion in the right superior parietal lobe, gyrus rectalis, and pons. Parietal hypoperfusion was correlated with morning stiffness and sleep disturbance and hypoperfusion of the gyrus rectalis with FIQ score, as previously mentioned. Hypoperfusion of the pons was also significantly more important in patients with depression. On the other hand, a significant blood flow increase in both hemithalami and basal ganglia and a decrease in both temporal, the left temporo-occipital, and the right occipital lobes were observed after treatment by amitriptyline (21). ${ }^{18}$ F-FDG PET studies are also discordant. Whereas Yunus et al. found no abnormalities between patients with fibromyalgia and healthy subjects (8), 1 study recently described an increase in limbic metabolism with concomitant symptomatic improvement (18).

Many experimental differences could explain this discrepancy. In particular, most of these studies were limited by a region-of-interest method. Voxel-based analysis allowed testing of whole-brain involvement without anatomic a priori knowledge. In addition, the difference with ${ }^{18} \mathrm{~F}$ FDG PET studies may be explained by a dissociation between perfusion and metabolism. It is then possible to have brain perfusion abnormalities without corresponding changes in glucose uptake, as previously reported in patients with chronic fatigue syndrome (22). Similarly, previous studies reported significant pharmacokinetic differences between radiotracer findings evaluating $\mathrm{rCBF}$. In 
Bilateral parietal perfusion (BA7)
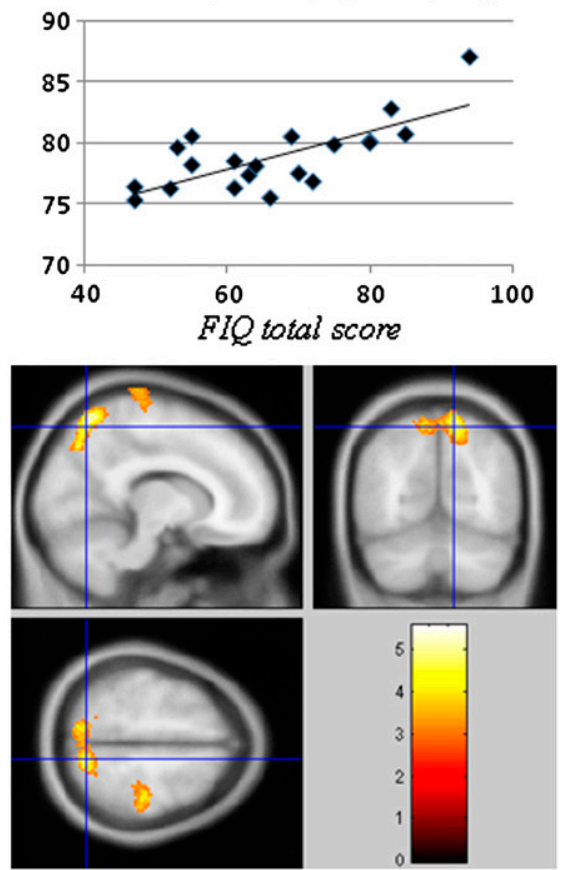

$r=0.6544 ; p=0.0043$
Right post-central perfusion (BA4)
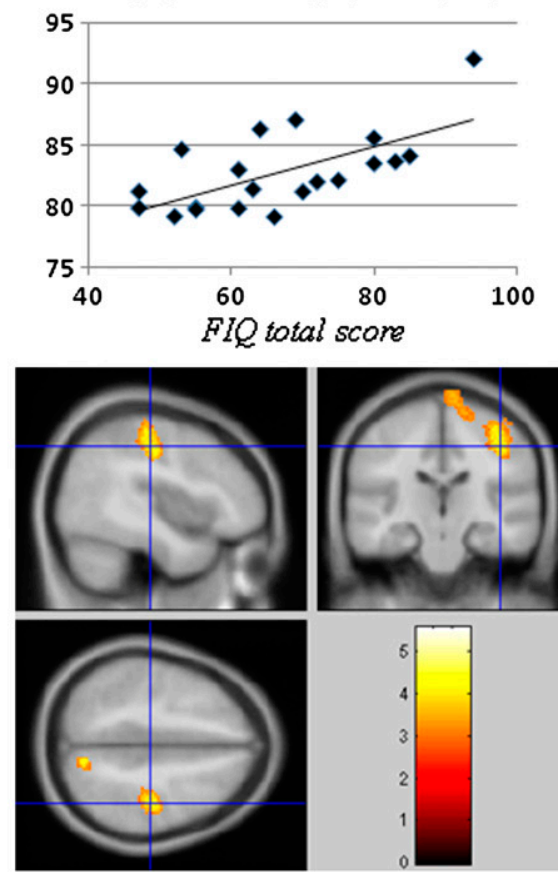

$$
\mathbf{r}=\mathbf{0 . 5 7 5 3 ; p}=\mathbf{0 . 0 1 2 2}
$$

Left anterior temporal perfusion
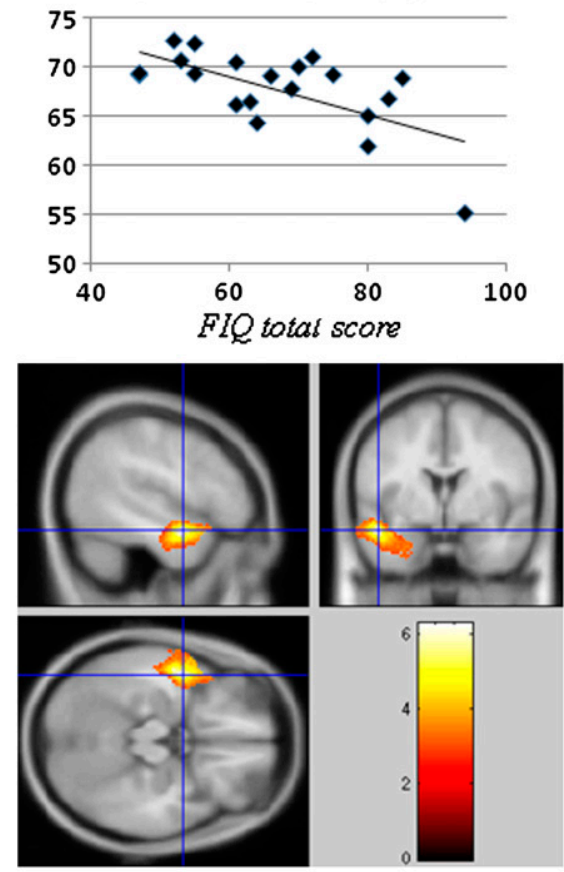

$\mathbf{r}=-\mathbf{0 . 5 6 1 7} ; \mathrm{p}=\mathbf{0 . 0 1 4 3}$

\section{Positive correlation}

\section{Negative correlation}

FIGURE 2. Anatomic localization of peaks of significant correlation between brain SPECT perfusion and FIQ total score $(P$ voxel $<$ 0.005 ; $P$ cluster $<0.05$, corrected for multiple comparisons; age as nuisance variable).

particular, ${ }^{99 \mathrm{~m}} \mathrm{Tc}$-ethylcysteinate dimer uptake is known to better reflect $\mathrm{rCBF}$ than ${ }^{99 \mathrm{~m}} \mathrm{Tc}-$ hexamethylpropyleneamine oxime in high-flow regions and to be more sensitive for the regional metabolic rate estimation, especially in the medial temporal cortex (23).

\section{CONCLUSION}

These results show that brain perfusion abnormalities in patients with fibromyalgia are independent of the patient's anxiety and depression status and correlate with the clinical severity of the disease, expressed by the disability and evaluated by the FIQ total score.

Because FIQ has been suggested as the most logical measure to evaluate functional status in clinical trials in fibromyalgia syndrome (24), these findings reinforce the concept of using brain perfusion SPECT to objectively evaluate therapeutic results in controlled studies $(14,25)$. In addition, as current pharmacologic and psychologic therapies act differently on both components of pain, this study suggests that a central effect could be expected in, respectively, the parietal cortex and the limbic system.

\section{ACKNOWLEDGMENTS}

Dr. Robert Coluzzi reviewed the English version of the manuscript. Bristol-Myers Squib sponsored the color reproduction of the figures.

\section{REFERENCES}

1. Walker JG, Littlejohn GO. Measuring quality of life in rheumatic conditions. Clin Rheumatol. 2007;26:671-673.

2. Desmeules JA, Cedraschi C, Rapiti E, et al. Neurophysiologic evidence for a central sensitization in patients with fibromyalgia. Arthritis Rheum. 2003;48:1420-1429.

3. Yunus MB. Role of central sensitization in symptoms beyond muscle pain, and the evaluation of a patient with widespread pain. Best Pract Res Clin Rheumatol. 2007;21:481-497.

4. Guedj E, Taieb D, Cammilleri S, et al. ${ }^{99 \mathrm{~m} T c-E C D}$ brain perfusion SPECT in hyperalgesic fibromyalgia. Eur J Nucl Med Mol Imaging. 2007;34:130-134.

5. Mountz JM, Bradley LA, Modell JG, et al. Fibromyalgia in women: abnormalities of regional cerebral blood flow in the thalamus and the caudate nucleus are associated with low pain threshold levels. Arthritis Rheum. 1995;38:926-938.

6. Mountz JM, Bradley LA, Alarcón GS. Abnormal functional activity of the central nervous system in fibromyalgia syndrome. Am J Med Sci. 1998;315: 385-396.

7. Kwiatek R, Barnden L, Tedman R, et al. Regional cerebral blood flow in fibromyalgia: single-photon-emission computed tomography evidence of reduction in the pontine tegmentum and thalami. Arthritis Rheum. 2000;43:2823-2833.

8. Yunus MB, Young CS, Saeed SA, Mountz JM, Aldag JC. Positron emission tomography in patients with fibromyalgia syndrome and healthy controls. Arthritis Rheum. 2004;51:513-518.

9. Wolfe F, Smythe HA, Yunus MB, et al. The American College of Rheumatology 1990 criteria for the classification of fibromyalgia. Report of the Multicenter Criteria Committee. Arthritis Rheum. 1990;33:160-172.

10. Boureau F, Luu M, Doubrère JF. Comparative study of the validity of four French McGill Pain Questionnaire (MPQ) versions. Pain. 1992;50:59-65.

11. Thieme K, Spies C, Sinha P, Turk DC, Flor H. Predictors of pain behaviors in fibromyalgia syndrome. Arthritis Rheum. 2005;53:343-350.

12. Zigmond AS, Snaith RP. The hospital anxiety and depression scale. Acta Psychiatr Scand. 1983;67:361-370.

13. White KP, Speechley M, Harth M, Ostbye T. Comparing self-reported function and work disability in 100 community cases of fibromyalgia syndrome versus controls in London, Ontario: the London Fibromyalgia Epidemiology Study. Arthritis Rheum. 1999;42:76-83. 
14. Guedj E, Cammilleri S, Colavolpe C, et al. Predictive value of brain perfusion SPECT for ketamine response in hyperalgesic fibromyalgia. Eur J Nucl Med Mol Imaging. 2007;34:1274-1279.

15. Gracely RH, Petzke F, Wolf JM, Clauw DJ. Functional magnetic resonance imaging evidence of augmented pain processing in fibromyalgia. Arthritis Rheum. 2002;46:1333-1343.

16. Gur A, Karakoc M, Erdogan S, Nas K, Cevik R, Sarac AJ. Regional cerebral blood flow and cytokines in young females with fibromyalgia. Clin Exp Rheumatol. 2002;20:753-760.

17. Cavada C, Company T, Tejedor J, Cruz-Rizzolo RJ, Reinoso-Suarez F. The anatomical connections of the macaque monkey orbitofrontal cortex: a review. Cereb Cortex. 2000;10:220-242.

18. Walitt B, Roebuck-Spencer T, Esposito G, et al. The effects of multidisciplinary therapy on positron emission tomography of the brain in fibromyalgia: a pilot study. Rheumatol Int. 2007;27:1019-1024.

19. Peyron R, Garcia-Larrea L, Gregoire MC, et al. Haemodynamic brain responses to acute pain in humans: sensory and attentional networks. Brain. 1999;122:1765-1780.
20. Casey KL. Forebrain mechanisms of nociception and pain: analysis through imaging. Proc Natl Acad Sci USA. 1999;96:7668-7674.

21. Adiguzel O, Kaptanoglu E, Turgut B, Nacitarhan V. The possible effect of clinical recovery on regional cerebral blood flow deficits in fibromyalgia: a prospective study with semiquantitative SPECT. South Med J. 2004;97:651-655.

22. Abu-Judeh HH, Levine S, Kumar M, et al. Comparison of SPET brain perfusion and ${ }^{18} \mathrm{~F}$-FDG brain metabolism in patients with chronic fatigue syndrome. $\mathrm{Nucl}$ Med Commun. 1998;19:1065-1071.

23. Hyun Y, Lee JS, Rha JH, Lee IK, Ha CK, Lee DS. Different uptake of ${ }^{99 m}$ TcECD and ${ }^{99 m}$ Tc-HMPAO in the same brains: analysis by statistical parametric mapping. Eur J Nucl Med. 2001;28:191-197.

24. White KP, Harth M. An analytical review of 24 controlled clinical trials for fibromyalgia syndrome (FMS). Pain. 1996;64:211-219.

25. Guedj E, Cammilleri S, Colavolpe C, de Laforte C, Niboyet J, Mundler O. Follow-up of pain processing recovery after ketamine in hyperalgesic fibromyalgia patients using brain perfusion ECD-SPECT. Eur J Nucl Med Mol Imaging. 2007;34:2115-2119. 